\title{
考虑表面形貌与受力变形的装配精度分析方法
}

\author{
刘检华 $^{1}$ 张志强 $^{2}$ 夏焕雄 $^{1}$ 巩 浩 $^{1}$ 邵 楠 $^{1}$ \\ (1. 北京理工大学机械与车辆学院 北京 100081 ; \\ 2. 北京卫星制造厂 北京 100094)
}

\begin{abstract}
摘要: 提高精度是机械制造学科永恒的追求目标之一, 随着精密机电产品不断追求更高精度, 装配精度分析的精确性要求也在 不断提高, 在此背景下, 提出综合考虑表面形貌与受力变形的装配精度分析方法。首先, 以非理想表面模型为基础, 通过小位 移旋量模型以及基函数线性叠加方法分别对定位/定向误差、形状误差进行建模; 并基于共轭梯度-快速傅里叶变换算法, 建立不 同类型非理想配合表面的接触变形计算方法; 然后, 以改进多面体模型进行装配偏差累积计算, 实现了综合考虑表面形貌与受 力变形的装配精度分析; 最后, 通过实例, 对比分析了是否采用非理想表面模型以及是否考虑受力变形条件下装配精度分析的 结果差异。数值研究结果表明, 所提方法能够提高装配精度分析的准确性, 从而为精密机械产品精度设计提供更为准确的指导。
\end{abstract} 关键词: 装配精度分析; 受力变形; 表面形貌; 公差分析; 公差设计

中图分类号: TH124

\section{Assembly Accuracy Analysis with Consideration of Form Defects and Surface Deformations}

\section{LIU Jianhua ${ }^{1}$ ZHANG Zhiqiang ${ }^{2}$ XIA Huanxiong ${ }^{1}$ GONG Hao ${ }^{1}$ SHAO Nan ${ }^{1}$}

(1. School of Mechanical Engineering, Beijing Institute of Technology, Beijing 100081;

2. Beijing Spacecraft, Beijing 100094)

\begin{abstract}
Improving accuracy is one of the permanently pursued goals of the mechanical manufacturing discipline. With the continuous pursuit of higher precision for precision eletromechanical products, the accuracy requirements of tolerance analysis are also continuously improved. Hence, a method for assembly accuracy analysis with consideration of form defects and surface deformations is proposed. First, the positioning / orientation errors and form error are modeled by a small displacement torsor model and a linear basis function superposition method, respectively, based on the skin model shapes. Then, the contact deformations of different types of non-ideal matching surfaces are calculated by applying a conjugate gradient-fast Fourier transform algorithm. Thereafter, a modified polytope model is used to calculate the cumulative assembly deviations to achieve assembly accuracy analysis with a combined consideration of form defects and surface deformations. Finally, the differences of the instances considering the form defects and surface deformations whether or not are compared. The numerical results indicate that the proposed method can improve the accuracy of assembly accuracy analysis and provide more accurate guidance for product tolerance design.
\end{abstract}

Key words: assembly accuracy analysis; surface deformations; form defects; tolerance analysis; tolerance design

\section{0 前言}

随着机电产品不断向小型化、复杂化、高精度、 性能高稳定等方向发展, 产品装配要求越来越高, 装配性能保证也越来越困难 ${ }^{[1]}$ 。几何精度设计是保 证产品性能的重要手段, 通过几何精度设计, 既要

* 国家自然科学基金(51935003)和北京市自然科学基金(3204054)资助项 目。20200610 收到初稿, 20201215 收到修改稿
控制零件表面的制造误差，保证零件的互换性和制 造的经济性, 也要满足整个产品的性能要求。

装配精度分析技术，主要用于验证产品设计阶 段的几何精度设计方案是否能够满足产品功能要 求 ${ }^{[2]}$ 。公差分析数学模型是装配精度分析的基础, REQUICHA 首先研究并给出了公差的数学定义, 以 构成几何特征的点的变动为基础, 提出了实体漂移 模型 ${ }^{[3]}$ 。矩阵模型(Matrix model) ${ }^{[4]}$ 通过齐次矩阵, 包括旋转和平移矩阵, 来表达几何特征在公差域内 
的相对位置。矢量环模型(Vector loop $)^{[5]}$ 采用直接线 性化方法(Direct linearization method, DLM), 通过将 几何公差引起的偏差转化为尺寸偏差, 并通过求导 的方式确定各偏差项的系数, 从而得到装配偏差线 性计算公式。小位移旋量模型(Small displacement torsor, SDT $)^{[6]}$ 将目标特征的变动分为转动和平动两 个部分, 采用两个三元矢量分别表示沿三个平动自 由度方向的偏差以及绕三个转动自由度方向的偏差。 公差变动矢量图模型(Tolerance map, T-Map $)^{[7]}$ 以旋 量表达为基础, 通过重心坐标进行建模, 模型中每 一个点对应着几何特征在公差域范围内的一种变动 情况。多面体模型(Polytope $)^{[8]}$ 通过离散化的几何特 征边界将公差约束范围定义为一系列不等式组, 形 成线性约束模型。

由于实际加工的零件表面不可避免地会与理想 几何表面存在差异, 随着产品精度要求不断提高, 把这些差异简单地处理成理想几何表面的平移和旋 转, 已经无法准确体现零件表面的形貌特征对装配 精度的影响。非理想表面模型(Skin model shape, SMS $)^{[9]}$ 提供了一种公差分析领域中的误差建模方 法, 该概念的提出是为了尽可能真实地体现零件表 面的几何误差。在产品设计阶段, 该模型依据产品 的公差设计生成满足公差规范的非理想表面模 型 ${ }^{[10]}$ 。而对于加工完成的零部件, 在有实测数据的 基础上, 非理想表面模型可以通过一些统计形状分 析(Statistical shape analysis, SSA)以及主成分分析 (Principal component analysis, PCA) 等 ${ }^{[11]}$ 方法进行建 模。在生成非理想表面模型的基础上, 公差仿真与 分析可以通过求解不同零件之间配合表面的相对位 置关系进行, 常用的求解相对位置关系的方法包括: 差表面法 ${ }^{[12]}$, 凸包法 ${ }^{[13]}$, 点云配准法 ${ }^{[14]}$ 等。

除了表面形貌会对装配精度分析结果产生影响, 在产品装配过程中, 由于定位夹紧等外力以及零部 件自身的重力等作用, 零件表面也会产生一定的变 形, 而这些变形又会进一步影响零件之间的相互位 置关系, 对产品最终的装配质量产生影响 ${ }^{[15]}$ 。在考 虑变形影响的装配精度分析研究方面, 以汽车车身 为代表的薄板类零件一直以来都是一个研究热点。 针对该类零件的变形计算, 有限元分析 (Finite element analysis, FEA)计算是一种最为直接的计算 方法 ${ }^{[16]}$, 但其计算效率不能满足快速计算的要求。 为解决该问题, 影响系数法 (Method of influence coefficients, MIC)仅通过一次有限元计算获得刚度 矩阵, 再通过刚度矩阵求解变形, 大大提高了受力 变形的计算效率 ${ }^{[17]}$ 。此外, 实际产品中依然大量存
在着一类有一定刚度 $($ 相对于薄板类弱刚性零件而 言)的实体类零件, 该类零件在外力作用下的变形计 算对于精度分析领域的研究来说依然是一个挑战。 有限元计算仍然是被广泛采用的一种计算方法, 即 通过有限元方法计算外力作用下的零部件变形, 并 用计算得到的变形数据进行公差分析 ${ }^{[18]}$ 。而在有限 元计算过程中采用何种模型，对装配精度分析结果 的准确性及计算效率就有着很大的影响, 采用理想 表面模型进行计算可能会与实际情况产生较大误 差 ${ }^{[19]}$, 而采用非理想表面模型进行计算虽然能够获 得较高的准确度, 但计算效率会大大降低 ${ }^{[20]}$ 。

综上所述, 现有装配精度分析方面的研究成果 仍未能较好地将表面形貌与受力变形进行耦合计算 分析。为此, 本文提出了综合考虑表面形貌与受力 变形的装配精度分析方法, 该方法通过基函数线性 叠加方法对非理想表面进行建模, 基于共轭梯度快速傅里叶变换算法计算非理想配合表面的接触变 形, 以改进的多面体模型进行装配偏差累积计算。 实例表明, 该方法能够提高装配精度分析的准确性。

\section{1 非理想表面模型建模方法}

现有的装配精度分析方法, 多数将零件表面的 加工误差简化为理想几何表面沿其理论位置的平移 和转动, 这种简化忽略了表面形貌对零件之间相互 位置的影响, 进而影响精度分析结果的准确性。为 此, 本节基于非理想表面建模技术，采用小位移旋 量模型对定位/定向误差进行建模, 采用基函数叠加 方法对形状误差进行建模, 并将二者叠加得到符合 公差规范要求的考虑表面形貌的非理想表面模型。

\section{1 定位与定向误差建模}

当几何特征被定位公差 $t_{p}$ 与定向公差 $t_{\mathrm{o}}$ 约束时, 定位与定向误差可以通过小位移旋量 (Small displacement torsor, SDT)表示, 包括一组表示沿着 $x-, y-, z-$ 轴平动的分量 $\left[\begin{array}{lll}\mu & v & \omega\end{array}\right]$ 与一组表示绕着 $x-, y-z-$-轴转动的分量 $\left[\begin{array}{lll}\alpha & \beta & \gamma\end{array}\right]$ 。常见几何特征的 小位移旋量表示及其取值范围如表 1 所示, 表 1 中, $a$ 和 $b$ 分别表示矩形平面的长和宽, $d$ 表示圆环面的 外径, $h$ 表示柱面的高度。

在此基础上, 包含定位/定向误差的几何表面通 过对理想位置下的几何表面进行平移旋转即可得到, 平移和旋转的数值大小通过给定的 SDT 参数确定, 这里用 $S_{p}$ 表示带有定位/定向误差的几何表面内离 散点的坐标值。 
表 1 常见几何特征的小位移旋量

\begin{tabular}{|c|c|c|c|}
\hline & 矩形平面 & 圆环面 & 柱面 \\
\hline 示意图 & $k_{x}^{y}$ & $\sqrt{\sqrt{\|\left[t_{p}|A| B\right.}}$ & $\underbrace{\frac{\varphi \phi t}{1} \phi t}_{A}$ \\
\hline $\begin{array}{l}\text { 小位移 } \\
\text { 旋量 }\end{array}$ & $\left\{S D T_{p p o}\right\}_{o}=\left[\begin{array}{cc}\alpha & 0 \\
\beta & 0 \\
0 & \omega\end{array}\right]$ & $\left\{S D T_{a p o}\right\}_{o}=\left[\begin{array}{cc}\alpha & 0 \\
\beta & 0 \\
0 & w\end{array}\right]$ & $\left\{S D T_{c p o}\right\}_{o}=\left[\begin{array}{cc}\alpha & u \\
\beta & v \\
0 & 0\end{array}\right]$ \\
\hline $\begin{array}{l}\text { 取值 } \\
\text { 范围 }\end{array}$ & $\alpha \in\left[-\frac{t_{p}}{b}, \frac{t_{p}}{b}\right] \quad \beta \in\left[-\frac{t_{p}}{a}, \frac{t_{p}}{a}\right] \quad \omega \in\left[-\frac{t_{p}}{2}, \frac{t_{p}}{2}\right]$ & $\alpha \in\left[\begin{array}{cc}-\frac{t_{p}}{d} & \frac{t_{p}}{d}\end{array}\right] \quad \beta \in\left[\begin{array}{cc}-\frac{t_{p}}{d} & \frac{t_{p}}{d}\end{array}\right] \quad w \in\left[\begin{array}{ll}-\frac{t_{p}}{2} & \frac{t_{p}}{2}\end{array}\right.$ & $\begin{array}{ll}\alpha \in\left[\begin{array}{cc}t_{p} & t_{p} \\
h & \frac{t_{p}}{h}\end{array}\right] & \beta \in\left[\begin{array}{cc}-\frac{t_{p}}{h} & t_{p} \\
h\end{array}\right] \\
u \in\left[\begin{array}{ll}-\frac{t_{p}}{2} & \frac{t_{p}}{2}\end{array}\right] & v \in\left[\begin{array}{ll}-\frac{t_{p}}{2} & \frac{t_{p}}{2}\end{array}\right]\end{array}$ \\
\hline $\begin{array}{l}\text { 约束 } \\
\text { 条件 }\end{array}$ & $\left|\omega \pm \alpha \cdot \frac{b}{2} \pm \beta \cdot \frac{a}{2}\right| \leqslant \frac{t_{p}}{2}$ & $\left|w \pm \frac{d}{2} \cdot \sqrt{\alpha^{2}+\beta^{2}}\right| \leqslant \frac{t_{p}}{2}$ & $\left(u \pm \frac{d}{2} \cdot \alpha\right)^{2}+\left(v \pm \frac{d}{2} \cdot \beta\right)^{2} \leqslant\left(\frac{t_{p}}{2}\right)^{2}$ \\
\hline
\end{tabular}

\section{2 形状误差建模}

与定位或定向公差类似，形状公差同样对几何 特征的变动范围做出限制, 以矩形平面为例, 其形 状公差域也为两平行平面之间的区域, 如图 1 所示。 通过形状误差建模所生成的非理想表面仍需要满足 形状公差的约束条件。

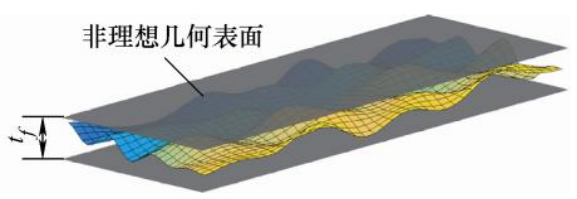

图 1 矩形平面的形状公差域示意图

形状误差可以通过一种基函数叠加的方式完成 建模, 采用的基函数依据不同的几何特征类型而不 同，该过程可以表示为

$$
S_{f}=\sum \lambda_{i} g_{i}
$$

式中, $S_{f}$ 表示带有形状误差的表面内离散点的坐标; $g_{i}$ 表示各个基函数; $\lambda_{i}$ 表示各个基函数的系数。

这种基函数叠加方法的一大优势是：在设计阶 段可以通过指定基函数系数的方式模拟形状误差, 同时, 如果已经有一批带有实测数据的样件, 可对 这些实测数据进行统计分析, 例如基于统计形状分 析法, 得到基函数系数的分布规律，进而更为真实 地模拟形状误差。需要说明的是, 在设计阶段根据 统计参量随机生成的基函数系数可能会使得生成的 带有形状误差的几何表面不能满足形状公差约束条 件, 因此, 在随机生成一组系数后, 要先进行形状 误差评定。若误差评定结果未能满足形状公差要求, 则需要对其进行修正，使其满足公差规范。

表 2 不同几何特征的基函数形式

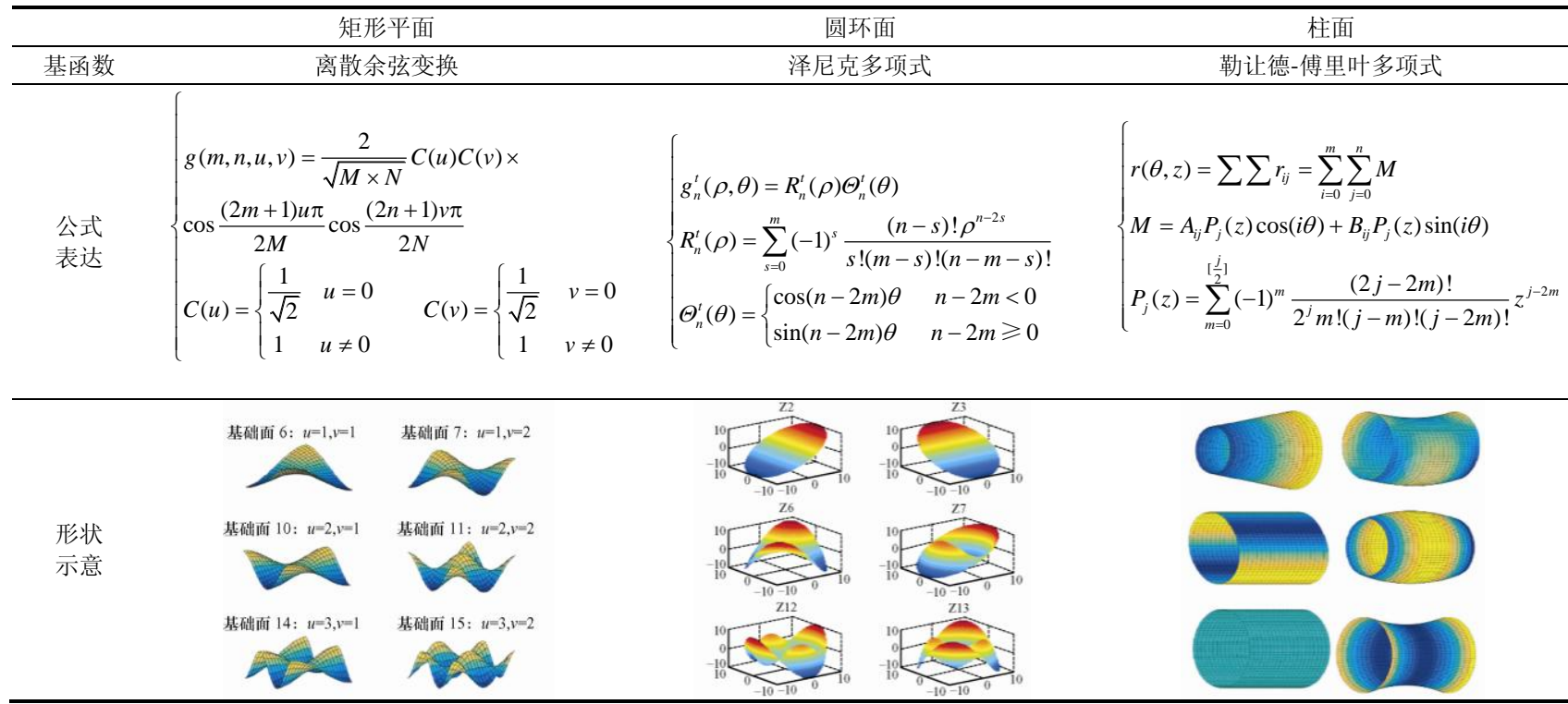


数; 采用泽尼克多项式(Zernike polynomials)作为圆 形平面和圆环形平面形状误差的基函数; 采用勒让 德-傅里叶(Legendre-Fourier, L-F)多项式作为柱面特 征形状误差的基函数。以上三类基函数表达式及其 对应的形状示意图如表 2 所示。表中离散余弦变换 表达式中, $m, n, u$ 和 $v$ 为变量, $M$ 和 $N$ 分别表示矩 形表面离散点在其长宽方向离散点的个数; 泽尼克 多项式中, $R_{n}^{t}(\rho)$ 为仅与径向有关的项, $\Theta_{n}^{t}(\theta)$ 为仅 与幅角有关的项, $n$ 为多项式的阶数, $t$ 为任意正或 负的整数, 其值恒与 $n$ 同奇偶性, $t \leqslant n$; 勒让德傅里叶多项式中, $r(\theta, z)$ 表示非理想柱面在 $(\theta, z)$ 处 的半径, $P_{j}(z)$ 为勒让德多项式, $A_{i j}$ 与 $B_{i j}$ 为傅里叶 多项式中的系数。

\section{3 非理想表面模型}

在定位与定向误差、形状误差建模的基础上, 非理想表面模型通过在包含定位定向误差的几何表 面上叠加一个随机生成的包含形状误差的几何表面 生成, 即

$$
S_{t}=S_{p}+S_{f}
$$

式中, $S_{t}$ 表示所生成非理想表面上各个离散点的 坐标。

需要说明的是, 二者的直接叠加可能导致最终 生成的几何表面无法满足公差的要求, 因此需要在 叠加之后, 判断生成的非理想表面是否满足公差规 范, 如果不能满足, 则需要重新生成。这里以矩 形平面为例, 其非理想表面模型的建模过程如图 2 所示。

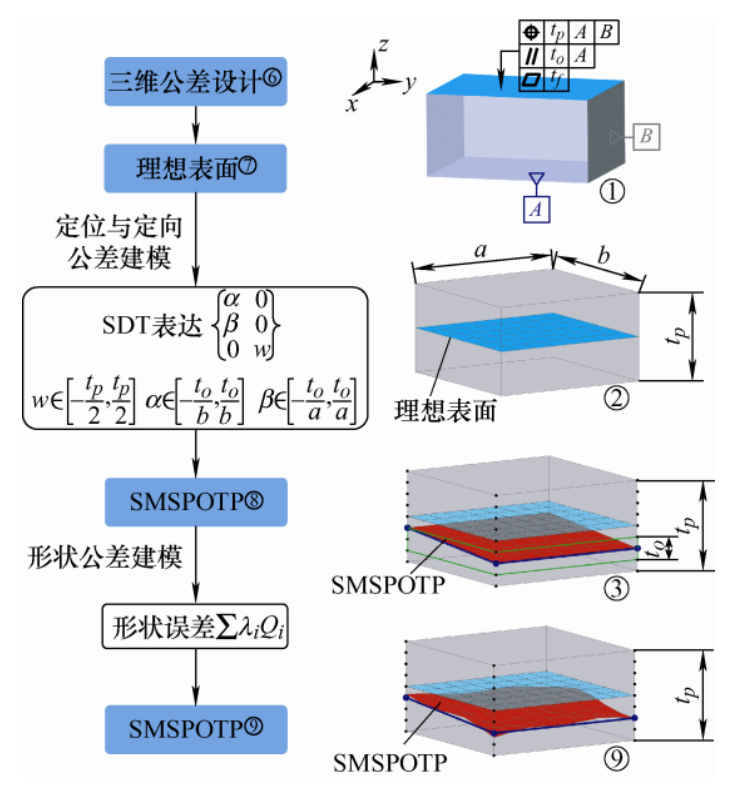

图 2 矩形平面的非理想表面模型建模流程

\section{2 受力变形计算方法}

除薄板类弱刚性零件外, 现有的装配精度分析 过程多数以刚体假设为前提, 对于产品装配过程中 可能出现的受力变形多数忽略不计, 而装配过程的 装夹力引起的零件变形, 尤其对精密机电产品而言, 有时会对最终的装配精度产生不可忽略的影响。目 前最常用的受力变形计算方法为有限元计算方法, 但是其计算效率低, 不适用于精度分析领域。边界 元方法(Boundary element method, BEM) 是广泛应用 于接触力学中的一种接触变形计算方法 ${ }^{[21]}$, 其计算 效率与准确性都能得到保证 ${ }^{[22]}$, 因此可以应用于装 配精度分析中受力变形的计算。其中, 共轭梯度快速傅里叶变换(Conjugate gradient-fast Fourier transform, CGFFT)计算方法 ${ }^{[23]}$ 是该领域的一种典型 计算方法，本节将采用该方法对两接触非理想表面 的变形量进行计算。

\section{1 共轭梯度-快速傅里叶变换算法}

第 2.1 节以两非理想矩形配合表面之间的接触 变形计算为例, 对共轭梯度-快速傅里叶变换算法进 行说明。根据接触力学的相关理论, 在弹性无摩擦 无粘假设下，任意两个非理想的矩形表面之间的接 触问题，可以等价为一个理想的表面与一个非理想 表面之间的接触 ${ }^{[24]}$, 如图 3 所示。

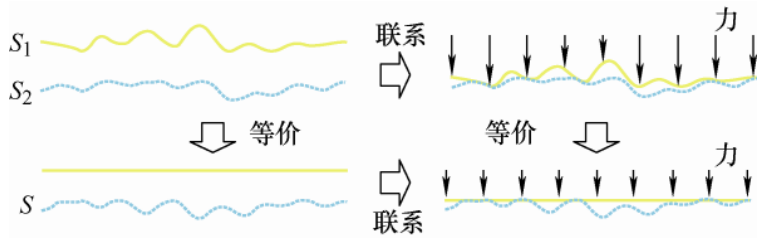

图 3 两非理想矩形配合表面等价为一理想表面与一非理想 表面之间的接触示意图

图 3 中, $S_{1}$ 与 $S_{2}$ 分别表示两接触的非理想表面, $S$ 表示等价之后的非理想表面, 上述表面均用离散 点进行表示，该等价过程用公式表示为

$$
S=S_{1}-S_{2}=S_{p 1}-S_{p 2}+\sum_{i=1}^{M \times N}\left(\lambda_{1 i}-\lambda_{2 i}\right) g_{i}
$$

在受外力作用下，该等价的非理想矩形表面各 个离散点的位移可以通过下式计算

$$
u_{i, j}=\sum_{k=1}^{M} \sum_{l=1}^{N} K_{i-k, j-l} p_{k, l}
$$

式中, $M$ 和 $N$ 分别表示矩形表面离散点在 $x$ 和 $y$ 方 向的个数; $p$ 表示每个离散点的接触力; $K$ 为影响 系数矩阵，其计算公式为 


$$
\begin{gathered}
K_{f, g}=\frac{2}{\pi E^{\prime}}\left[y_{m} \lg \left(x_{m}+\sqrt{x_{m}^{2}+y_{m}^{2}}\right)+x_{m} \lg \left(y_{m}+\sqrt{x_{m}^{2}+y_{m}^{2}}\right)-\right. \\
y_{m} \lg \left(x_{p}+\sqrt{x_{p}^{2}+y_{m}^{2}}\right)-x_{p} \lg \left(y_{m}+\sqrt{x_{p}^{2}+y_{m}^{2}}\right)- \\
y_{p} \lg \left(x_{m}+\sqrt{x_{m}^{2}+y_{p}^{2}}\right)-x_{m} \lg \left(y_{p}+\sqrt{x_{m}^{2}+y_{p}^{2}}\right)+ \\
\left.y_{p} \lg \left(x_{p}+\sqrt{x_{p}^{2}+y_{p}^{2}}\right)+x_{p} \lg \left(y_{p}+\sqrt{x_{p}^{2}+y_{p}^{2}}\right)\right]
\end{gathered}
$$

式中 , $x_{m}=x_{f}+\Delta x / 2, x_{p}=x_{f}-\Delta x / 2$, $y_{m}=y_{g}+\Delta y / 2, y_{p}=y_{g}-\Delta y / 2, \Delta x$ 和 $\Delta y$ 分别表 示相邻两离散点之间在 $x$ 和 $y$ 方向的距离; $E^{\prime}$ 为复 合弹性模量, 其计算公式为

$$
\frac{2}{E^{\prime}}=\frac{1-v_{1}^{2}}{E_{1}}+\frac{1-v_{2}^{2}}{E_{2}}
$$

式中, $E_{1}$ 和 $E_{2}$ 分别表示两接触零件的弹性模量; $v_{1}$ 和 $v_{2}$ 分别表示其泊松比。

为了保证两接触表面之间没有穿透以及矩形表 面内的离散点始终受压力, 存在以下约束条件

$$
c_{i, j} \geqslant 0 \quad p_{i, j} \geqslant 0
$$

式中, $c_{i, j}$ 表示对应离散点之间的间距; $p_{i, j}$ 表示离 散点的接触力。

如图 4 所示, 在接触区域, 有边界条件 $c_{i, j}=0$, $p_{i, j} \geqslant 0$; 在非接触区域, 有边界条件: $c_{i, j}>0$, $p_{i, j}=0$ 。因此, $c_{i, j}$ 和 $p_{i, j}$ 不能同时为非零实数, 即 满足如下条件

$$
c_{i, j} p_{i, j}=0
$$

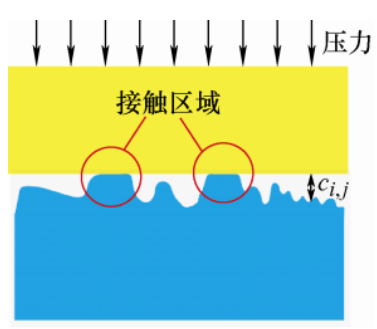

图 4 接触区域示意图

由最小势能原理, 该接触问题可以转化为一个 条件极值问题

$$
\begin{array}{ll}
\min & W(\boldsymbol{p})=\boldsymbol{c}^{\mathrm{T}} \boldsymbol{p}+\frac{1}{2} \boldsymbol{p}^{\mathrm{T}} \boldsymbol{K} \boldsymbol{p} \\
\text { s.t. } & c_{i, j} \geqslant 0, p_{i, j} \geqslant 0, c_{i, j} p_{i, j}=0
\end{array}
$$

在式(9)中, 最终两接触面之间的间隙 $c_{i, j}$ 取决 于计算得到的接触变形量 $u_{i, j}$, 二者之间的关系可以 表示为

$$
c_{i, j}=h_{i, j}+u_{i, j}
$$

式中, $h_{i, j}$ 表示两接触的非理想矩形表面之间的初始 间隙。

CG-FFT 计算方法即用来求解该条件极值问题, 其中, 共轭梯度法用来求解优化问题的搜索方向与 步长, 快速傅里叶变换方法用来快速求解卷积问题, 即式(4)。该计算方法能够实现局部受力变形的快速 准确计算。一个典型的计算结果如图 5 所示, 接触 点为图中黑色圆点所示, 由于受到外力作用, 接触 区域为很多被压平的小平面。

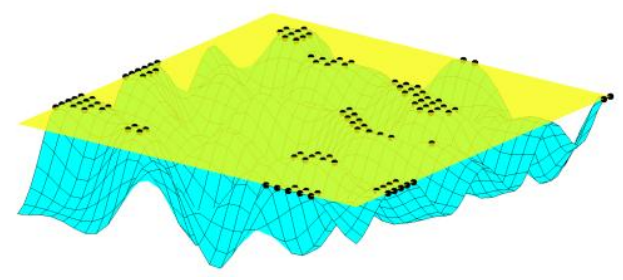

图 5 矩形平面 CG-FFT 计算接触区域示意图

\section{2 圆环面特征的变形计算}

CG-FFT 方法适用于矩形平面特征在外力作用下 的变形计算, 实际产品中, 存在大量由螺栓连接的法 兰面, 为了能够实现这类圆环面之间接触变形的快速 计算, 提出两种圆环面变形计算方法: ATR-E 与 ATR-T, 前者将圆环面展开为矩形平面, 而后者将圆 环面扩充为矩形平面。圆形平面可以看作是一类特殊 的圆环面，因此其受力变形计算方法与圆环面相同。

(1) ATR-T 算法: 该方法的基本原理是将圆环面 展开为一个长条状的矩形平面。如图 6 所示, 图 $6 \mathrm{a}$ 中的圆环被展开为图 $6 \mathrm{~b}$ 所示长为 $\pi(R+r)$, 宽为 $R-r$ 的细长型矩形。同时该矩形内的采样点沿着其 长、宽方向均为均匀分布。对如图 $6 \mathrm{~d}$ 所示的考虑表 面形貌的圆环特征, 其转化为矩形面如图 6e 所示。 在完成该转化过程后，便可采用 CG-FFT 算法进行 受力变形的计算。

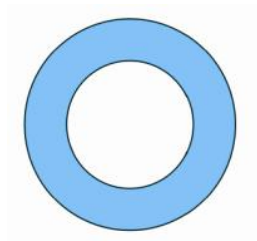

(a) 圆环面示意图

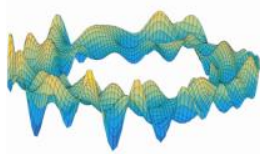

(d) 非理想几何圆环面

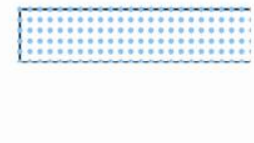

(b) 展开后的矩形面

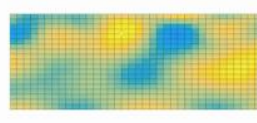

(e) 展开后的矩形面

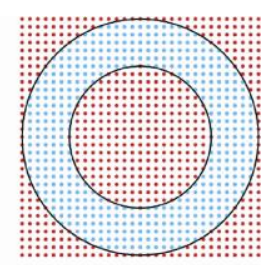

(c) 扩充后的矩形面

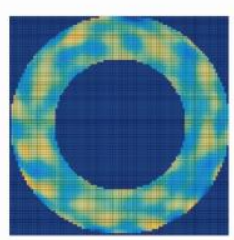

(f) 扩充后的矩形面
图 6 圆环面受力变形算法示意图 
(2) ATR-T 算法: 该方法的基本原理是将圆环面 扩充为一矩形平面。如图 6 所示, 图 6a 中的圆环被 扩充为图 6c 所示的正方形, 该正方形内的采样点沿 着其长、宽方向均匀分布。对如图 $6 \mathrm{~d}$ 所示的考虑表 面形貌的圆环特征, 其扩充为矩形面如图 6f 所示。 在完成该转化过程后, 便可采用 CG-FFT 算法进行 受力变形的计算。

对于第一种计算方法, 即 ATR-T 算法, 通过
CG-FFT 算法对转换后的矩形表面进行接触变形计 算, 得到变形后的矩形表面如图 7a 所示, 将该变形 后的矩形表面再一次转换为圆环面, 如图 7b 所示, 图 7c 为其俯视图。对于第二种计算方法, 即 ATR-E 算法, 通过 CG-FFT 算法对扩充后的矩形表面进行 接触变形计算, 得到变形后的矩形表面如图 7d 所示, 将该变形后的矩形表面再一次转换为圆环面，如图 $7 \mathrm{e}$ 所示, 图 7f 为其俯视图。

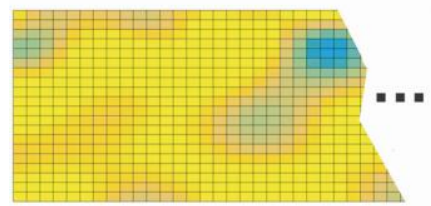

(a) 展开后的矩形表面

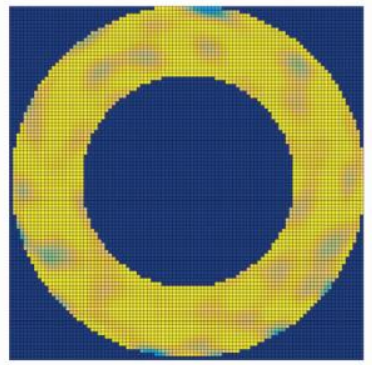

(d) 扩充后的矩形表面

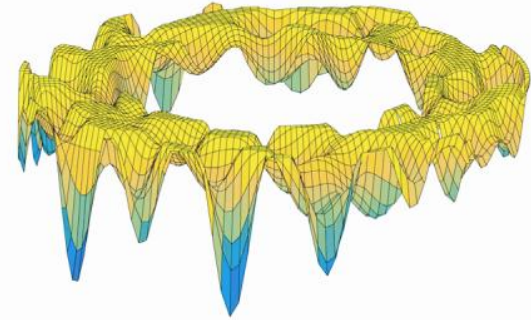

(b) ATR-T计算结果-3D

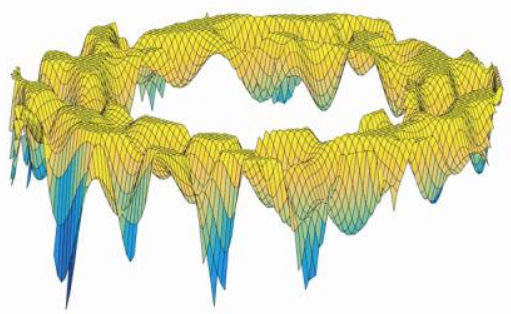

(e) ATR-E计算结果-3D

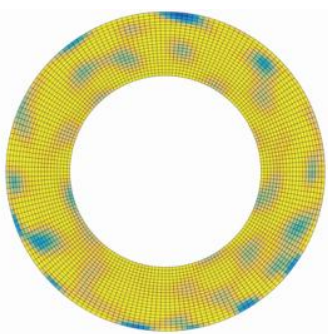

(c) ATR-T计算结果-俯视图

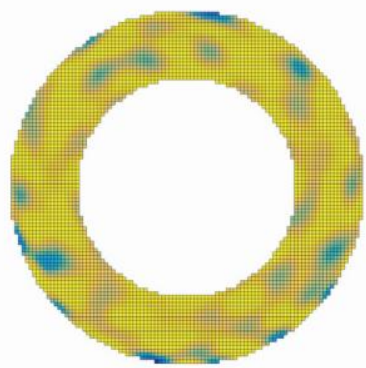

(f) ATR-E计算结果-俯视图

图 7 ATR-T 与 ATR-E 计算结果对比

直观地对图 7b、7c、7e、7f 中的计算结果进行 比较, 能够看出两种计算方法得到的结果大致相同。 为了进一步通过定量数据对两者的计算结果进行对 比, 分别计算两种计算方法下的非理想圆环面的变 形量, 即变形前后圆环面上最高点的高度差。两种 计算方法下, 对于图中给定的算例, 该变形量计算 结果分别为 $0.045 \mathrm{~mm}$ 与 $0.046 \mathrm{~mm}$, 二者的差别不 到 $2.5 \%$ 。同时由于两种计算方法下的数据点采样方 法不同, 因此 $2.5 \%$ 的差距足以证明这两种计算方法 均适用于圆环面的受力变形计算。

\section{3 柱面特征的变形计算}

对于相互配合的柱面特征, 其配合状态可能存 在两种情况, 即过盈配合与间隙配合。因此, 在随 机生成一组相互配合的非理想柱面后, 首先需要判 断其为过盈配合还是间隙配合。对于过盈配合, 则 其在装配过程中必然借助外力作用达到最终配合状 态。对于间隙配合, 其相对位置需要根据具体的装 配情况进行分析。图 8 所示为间隙配合状态下柱面 特征的受力变形计算方法示意图, 首先依据点云配 准方法 ${ }^{[14]}$ 求得两非理想柱面在不受外力作用下的
初始接触状态, 其次, 将上述初始接触区域附近的 非理想几何柱面展开为矩形(即将该问题等价为一 非理想矩形表面与一理想矩形表面之间的接触问 题), 针对该展开后的非理想矩形表面进行 CG-FFT 计算, 最终将计算得到的变形结果作为初始接触区 域附近的受力变形结果。

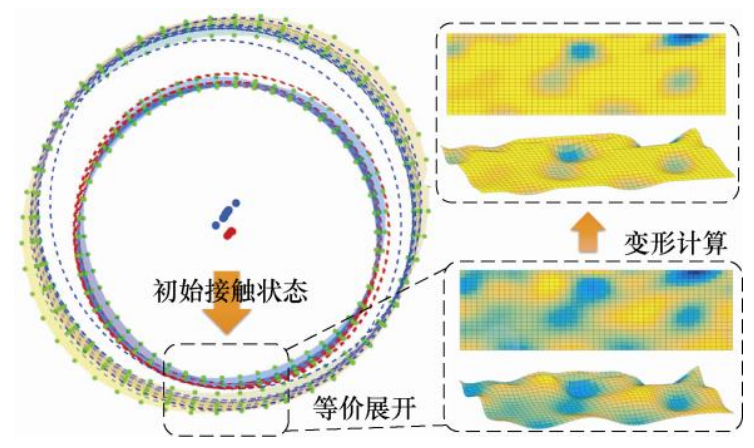

图 8 间隙配合状态下柱面接触变形计算示意图

\section{3 基于多面体模型的装配精度分析}

装配体由两个或两个以上零件按照一定的装配 
关系连接而成, 每个零件又由多个几何特征组成。 装配精度分析需要实现对每个零件的几何特征偏差 以及零件之间的配合偏差进行累积计算。本节提出 了基于改进多面体模型的装配精度分析方法, 实现 综合考虑表面形貌与受力变形的精度分析。

\section{1 多面体模型}

多面体模型将几何特征面离散为一系列点, 对 这些离散点进行约束, 使其处于公差约束或装配约 束的范围内 ${ }^{[8]}$ 。以公差约束为例, 几何特征上的点 需处于公差域内, 而实际加工的零件表面不可避免 的存在误差, 因此实际表面与理想表面必然不会完 全相同。此时, 实际表面上的离散点的约束则由该 点处的上下偏差数值以及该几何特征的公差数值同 时决定。以图 9 所示几何特征为例, $S$ 表示理想表 面, 而 $S_{0}$ 为其实际表面, 给定公差值的公差域为 $T Z$, $S_{0}$ 能够在公差域内变动, 即位于 $d_{i}^{\text {inf }}$ 与 $d_{i}^{\text {sup }}$ 之间。上 述约束对于实际表面内的任意一点 $N_{i}$ 可以表示为

$$
N_{i} \in S_{0}: d_{i}^{\text {inf }} \leqslant \varepsilon_{N_{i}} \cdot \boldsymbol{n}_{i} \leqslant d_{i}^{\text {sup }}
$$

式中, $\varepsilon_{N_{i}}$ 表示 $N_{i}$ 点处的变动; $\boldsymbol{n}_{i}$ 表示 $N_{i}$ 点处的 法向。

根据小位移旋量理论，上式可以在空间任意一 点 $M$ 处进行表达

$$
d_{i}^{\text {inf }} \leqslant\left(N_{i} M \times n_{i}\right) \cdot \boldsymbol{R}+\boldsymbol{n}_{i} \cdot \boldsymbol{T}_{M} \leqslant d_{i}^{\text {sup }}
$$

式中, $\boldsymbol{R}=(\alpha, \beta, \gamma)$, 表示转动分量; $\boldsymbol{T}_{M}=(u, v, w)$, 表示平动分量。

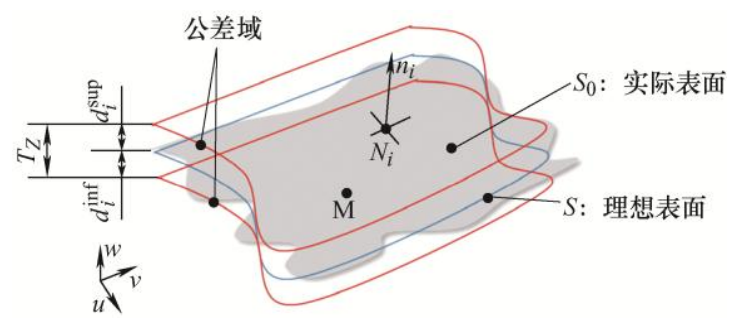

图 9 实际表面与公差域示意图

若设定 $\boldsymbol{N}_{i} \boldsymbol{M}=\left(d_{i u}, d_{i v}, d_{i w}\right), \boldsymbol{n}_{i}=\left(n_{i \alpha}, n_{i \beta}, n_{i \gamma}\right)$ 且 $\alpha=x_{1}, \quad \beta=x_{2}, \quad \gamma=x_{3}, \quad u=x_{4}, \quad v=x_{5}, \quad w=x_{6}$, 式(12)展开为

$$
\begin{aligned}
& d_{i}^{\mathrm{inf}} \leqslant\left(n_{i \beta} d_{i w}-n_{i \gamma} d_{i v}\right) x_{1}+\left(n_{i \gamma} d_{i u}-n_{i \alpha} d_{i w}\right) x_{2}+ \\
& \left(n_{i \alpha} d_{i v}-n_{i \beta} d_{i u}\right) x_{3}+n_{i \alpha} x_{4}+n_{i \beta} x_{5}+n_{i \gamma} x_{6} \leqslant d_{i}^{\text {sup }}
\end{aligned}
$$

因此, 对于 $S_{0}$ 内任意一点, 均有上述约束方程, 该方程实际上代表了六维空间内两相互平行的半空 间 ${ }^{[8]}$, 这两个半空间可以表示为

$$
\begin{aligned}
\bar{H}^{+}=\{ & \left\{\left(n_{i \beta} d_{i w}-n_{i \gamma} d_{i v}\right) x_{1}+\left(n_{i \gamma} d_{i u}-n_{i \alpha} d_{i w}\right) x_{2}+\right. \\
& \left.\left(n_{i \alpha} d_{i v}-n_{i \beta} d_{i u}\right) x_{3}+n_{i \alpha} x_{4}+n_{i \beta} x_{5}+n_{i \gamma} x_{6} \geqslant d_{i}^{\text {inf }}\right\} \\
\bar{H}^{-}= & \left\{\left(n_{i \beta} d_{i w}-n_{i \gamma} d_{i v}\right) x_{1}+\left(n_{i \gamma} d_{i u}-n_{i \alpha} d_{i w}\right) x_{2}+\right. \\
& \left.\left(n_{i \alpha} d_{i v}-n_{i \beta} d_{i u}\right) x_{3}+n_{i \alpha} x_{4}+n_{i \beta} x_{5}+n_{i \gamma} x_{6} \leqslant d_{i}^{\text {sup }}\right\}
\end{aligned}
$$

调整上式中 $\bar{H}^{+}$的符号, 式中半空间可统一表 达为

$$
\bar{H}^{-}: b_{i}+a_{i 1} x_{1}+\cdots+a_{i 6} x_{6} \leqslant 0
$$

对于 $S_{0}$ 内任意一点, 能够得到两个如式(15)所 示半空间, 则对于 $S_{0}$ 内的 $m$ 个离散点, 能够得到 $2 m$ 个半空间, 这些半空间的交集即为该实际表面的 多面体模型 ${ }^{[8]}$, 表示为

$$
P=\bigcap_{i=1}^{2 m} \bar{H}_{i}^{-}
$$

\section{2 综合考虑表面形貌与受力变形的多面体模型}

传统的多面体模型将几何特征看作是理想表 面, 忽略了表面形貌与受力变形的影响, 本小节 对传统的多面体模型进行改进, 将表面形貌及受 力变形的影响纳入到多面体模型的生成过程中。 多面体模型包括两大类：几何多面体模型与接触 多面体模型, 前者对单一表面受到的公差约束进 行建模, 后者对配合表面之间的装配约束进行 建模。

当几何特征被看作为理想表面时，其上下边界 $d_{i}^{\mathrm{inf}}$ 与 $d_{i}^{\text {sup }}$ 理论上仅与公差数值相关; 当考虑表面形 貌时, 实际表面上每一个离散点处的上下边界除了 与公差数值相关外, 还与该点处的上下偏差值相关; 当综合考虑表面形貌与受力变形时, 其上下边界值 则与变形后实际表面的上下偏差值和公差数值相关。 以矩形平面的几何多面体模型为例, 其理想表面的 多面体模型由 8 个半空间求交得到, 为一八面体; 当考虑表面形貌时, 首先生成其非理想表面模型, 通过该模型即能够得到每个离散点处的上下偏差数 值, 结合公差数值即可得到两倍于离散点数目的半 空间, 这些半空间的交集即为非理想矩形表面的多 面体模型; 当综合考虑表面形貌与受力变形时, 首 先求得变形后的非理想矩形表面, 此时能够得到一 组新的各离散点处的上下偏差数值, 结合公差数值, 即可得到每个离散点处的半空间表达式, 各个离散 点处半空间的交集即为综合考虑表面形貌与受力变 形的矩形平面多面体模型。以上不同条件下矩形平 面多面体模型示意图如图 10 所示。 


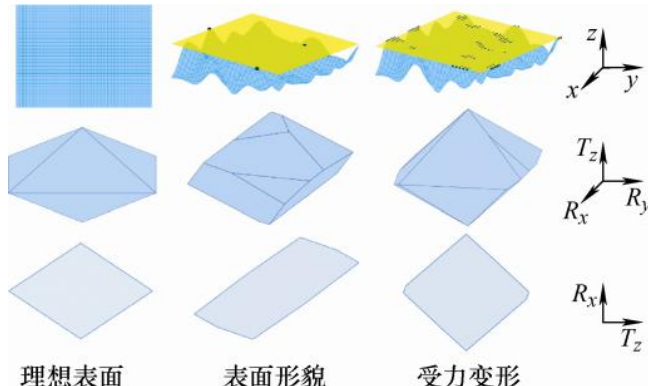

图 10 矩形表面的多面体模型示意图

几何多面体模型对受到公差约束的单一几何特 征进行建模，而接触多面体模型则对配合表面之间 的接触约束进行建模。同样地, 接触约束定义了相 互配合的特征之间允许的变动范围, 不同的是其约 束边界 $d_{i}^{\text {inf }}$ 与 $d_{i}^{\text {sup }}$ 会有所不同。以轴孔配合为例, 其 接触约束边界 $d_{i}^{\mathrm{inf}}$ 与 $d_{i}^{\text {sup }}$ 的大小取决于两柱面之间 的间隙。

\section{3 基于多面体模型的装配精度分析流程}

将多面体模型应用于装配精度分析, 需要对每 个多面体模型之间进行累积计算, 多面体模型之间 的运算方法包括求闵科夫斯基和与求交两种, 这两 种运算方法同时也对应了串联与并联两种不同的装 配结构, 示意图如图 11 所示 ${ }^{[25]}$ 。

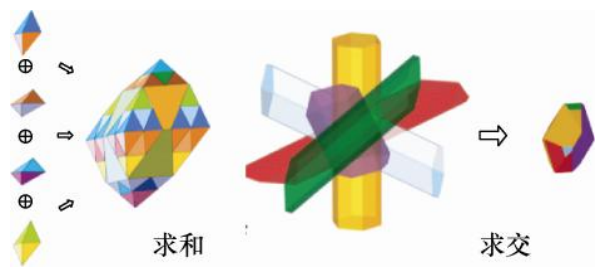

图 11 多面体模型的求和与求交运算示意图

基于多面体模型的装配精度分析流程如图 12 所示, 主要包括以下步骤。

(1) 根据装配体三维设计图纸, 理清其公差设 计方案以及装配约束关系, 整理与公差设计及装配 约束相关的几何特征。

（2）根据装配体的功能要求, 结合公差约束与 装配约束, 获得装配关系图, 从装配关系图中得到 功能要求的装配偏差传递路径。

(3) 逐一求解偏差传递路径上对应的多面体模 型。首先依据公差要求生成满足条件的非理想表面, 其次, 依据接触变形求解算法计算变形后的非理想 表面, 最终通过修改约束方程的上下边界参数得到 对应的几何多面体或接触多面体模型。

（4）依据装配偏差传递路径, 对于其上的串联结 构, 则对相应的多面体进行求闵科夫斯基和运算, 对 于其上的并联结构, 则对相应的多面体进行求交运算,
最终计算得到的多面体即为装配功能要求多面体。

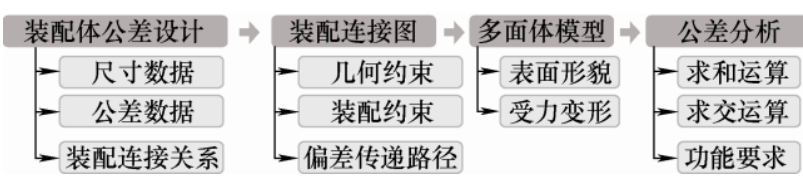

图 12 基于多面体模型的装配精度分析流程

\section{4 实例分析}

\section{1 研究实例}

HOMRI 等 $^{[8]}$ 首先提出了采用多面体模型进行 装配精度分析的流程，但其研究将几何特征看作是 理想表面并基于刚性体假设进行。本文在其基础上 丰富了多面体模型的应用条件与范围，为与其计算 结果进行对比，本节采用其论文中的相同实例进行 分析。所采用装配体实例如图 13 所示, 该装配体包 括三个零件：一组轴孔配合零件及一个底座，装配 功能要求为轴特征上 $M$ 点相对于底座的位置关系, 同时，本节中的多面体模型均将以图中的 $M$ 点为基 准点求解约束方程, 基准点可以任意选取, 且选取 不同的基准点不会影响最终的精度分析结果。各个 零件的详细公差设计如图 14 所示, 同时, 图中也对 每个零件的各个面进行了标记, 标号 $i . j$ 代表第 $i$ 个 零件的第 $j$ 个表面。

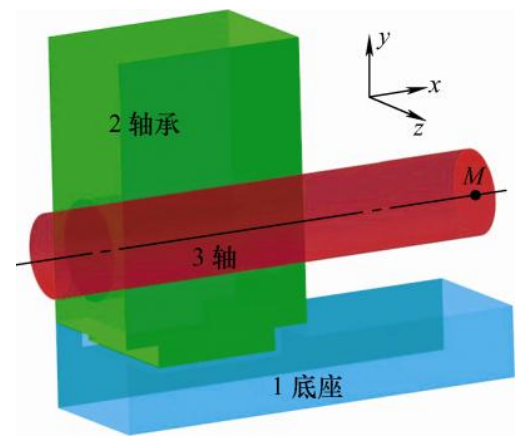

图 13 研究实例

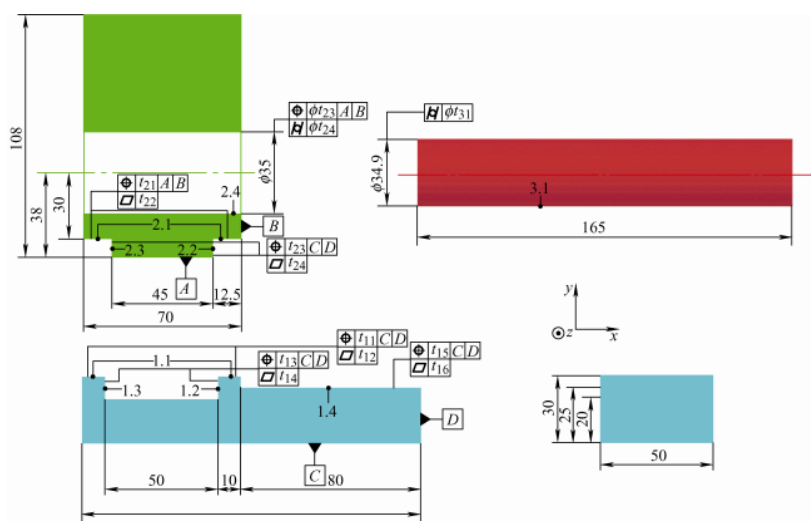

图 14 详细公差设计 
由该装配体的装配约束关系及公差设计方案, 能够得到其装配关系图, 如图 15 所示, 图中 $\mathrm{CP}$ 表 示柱面配合, PP 表示平面配合, FR 为装配功能要 求。从图中可以看出该装配体存在八个与公差约束 相关的几何特征, 分别为 $(1.0,1.1),(1.0,1.2),(1.0$, $1.3),(1.0,1.4),(2.0,2.1),(2.0,2.2),(2.0,2.3)$, $(2.0,2.4)$, 对应八个几何多面体; 同时, 表面 1.1 与 $2.1,1.2$ 与 $2.2,1.3$ 与 2.3 构成平面接触对, 表面 2.4 与 3.1 构成柱面接触对, 对应四个接触多 面体。这里用 $P_{i, j} / u, v$ 表示每个对应的多面体, 下标 与该多面体对应的几何特征相同, 例如 $P_{2.4 / 3.1}$ 表 示此例中的柱面接触多面体, 同理, 功能要求多 面体表示为 $P_{3.1 / 1.4}$ 。

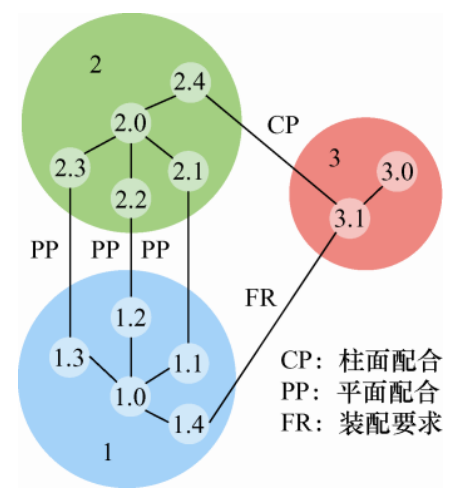

图 15 研究实例的装配连接图

由图 15 所示的装配连接图, 可以得到三条 偏差传递路径, 分别为: $1.4 \rightarrow 1.0 \rightarrow 1.3 \rightarrow 2.3 \rightarrow 2.0$ $\rightarrow 2.4 \rightarrow 3.1,1.4 \rightarrow 1.0 \rightarrow 1.2 \rightarrow 2.2 \rightarrow 2.0 \rightarrow 2.4 \rightarrow 3.1$, $1.4 \rightarrow 1.0 \rightarrow 1.1 \rightarrow 2.1 \rightarrow 2.0 \rightarrow 2.4 \rightarrow 3.1$ 。其中, 每条
传递路径中的 $1.4 \rightarrow 1.0$ 与 $2.0 \rightarrow 2.4 \rightarrow 3.1$ 为串联 部分, 进行装配精度分析时, 对应的多面体应进 行求和运算; 其余部分为并联部分, 进行精度分 析时, 每偏差传递路径上对应的多面体先进行求 和运算, 然后对得到的三个多面体进行求交运算。 这里将求解功能多面体 $P_{3.1 / 1.4}$ 时的三条并联偏 差传递路径对应的多面体模型运算结果记为 $P_{2.0 / 1.0}$

$$
\begin{gathered}
P_{2.0 / 1.0}=\left(P_{2.0 / 2.3} \oplus P_{2.3 / 1.3} \oplus P_{1.3 / 1.0}\right) \cap \\
\left(P_{2.0 / 2.2} \oplus P_{2.2 / 1.2} \oplus P_{1.2 / 1.0}\right) \cap \\
\left(P_{2.0 / 2.1} \oplus P_{2.1 / 1.1} \oplus P_{1.1 / 1.0}\right)
\end{gathered}
$$

然后, 对串联部分进行求和运算, 即可得到功 能要求多面体

$$
P_{3.1 / 1.4}=P_{3.1 / 2.4} \oplus P_{2.4 / 2.0} \oplus P_{2.0 / 1.0} \oplus P_{1.0 / 1.4}
$$

\section{2 未考虑受力变形的精度分析结果}

本节基于理想表面模型, 对未考虑受力变形的 装配精度分析结果进行求解, 即采用传统的多面体 模型, 在多面体模型生成过程中不考虑表面形貌与 受力变形的影响。分析时, 图 14 中定位公差数值均 为 $0.04 \mathrm{~mm}$, 形状公差数值均为 $0.02 \mathrm{~mm}$ 。对于式(17), 通过对应的求和与求交运算即可求得多面体 $P_{2.0 / 1.0}$, 该过程如图 16 所示。

在得到多面体 $P_{2.0 / 1.0}$ 后, 依据式(18), 对多面 体 $P_{3.1 / 2.4}, P_{2.4 / 2.0}, P_{2.0 / 1.0}$ 与 $P_{1.0 / 1.4}$ 进行求和运算 即可得到功能要求多面体 $P_{3.1 / 1.4}$, 该过程如图 17 所示。

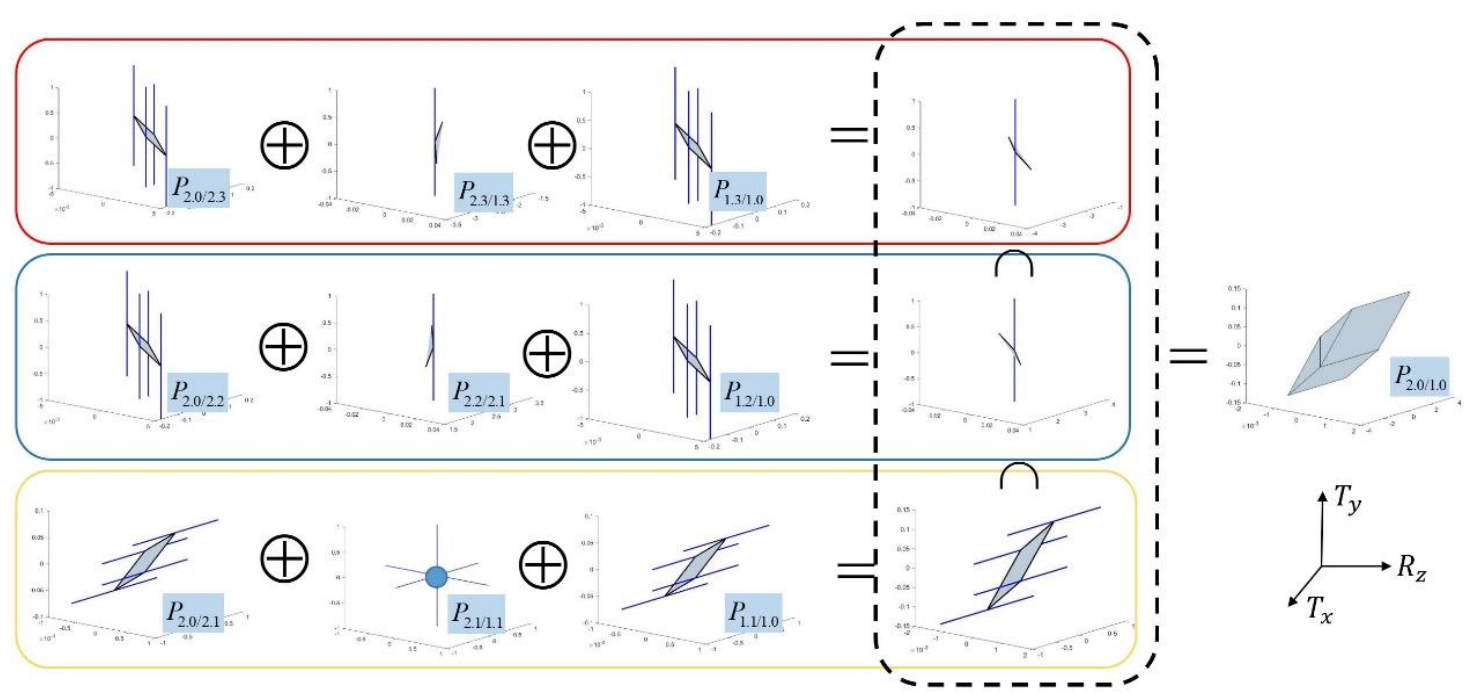

图 16 多面体 $P_{2.0 / 1.0}$ 三维示意图 


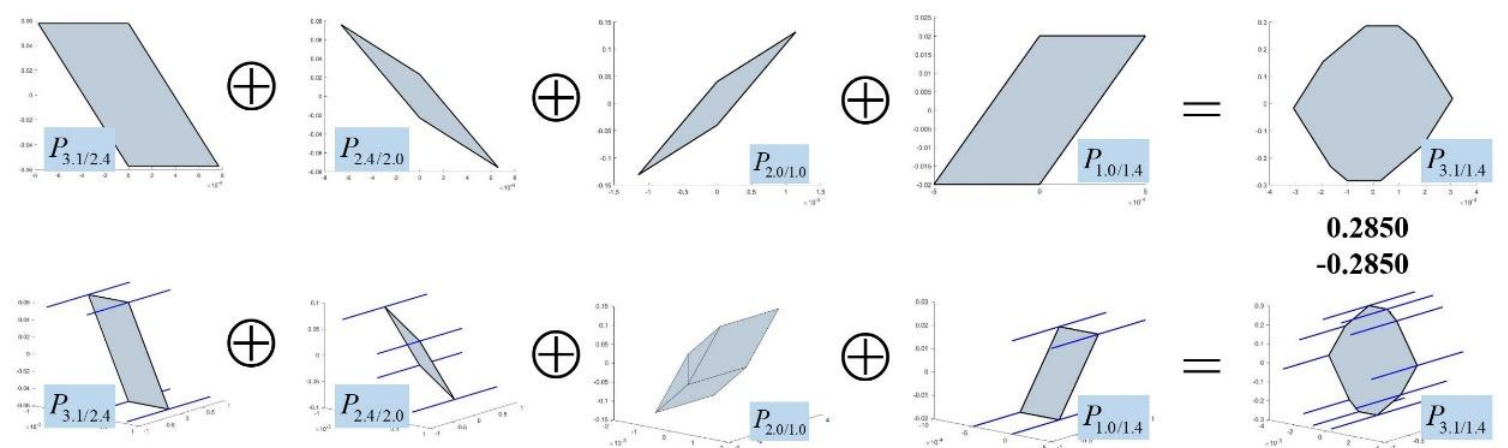

图 17 面体 $P_{3.1 / 1.4}$ 计算过程

\section{3 考虑受力变形的精度分析结果}

本小节基于非理想表面模型, 对考虑变形的装 配精度分析结果进行求解, 即采用综合考虑表面形 貌与受力变形的多面体模型进行分析。首先, 生成 该装配体中各个相关表面的非理想表面模型, 如 图 18 所示。

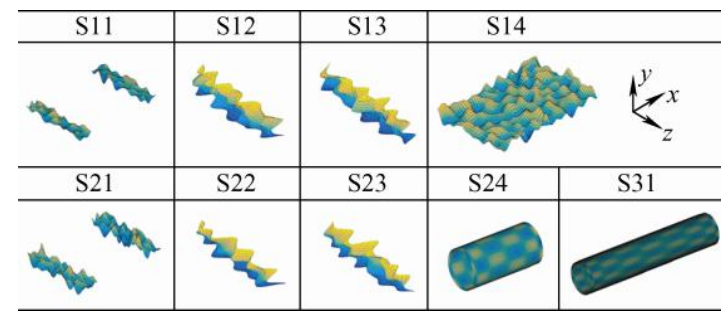

图 18 各几何特征的非理想表面

对于本节中的研究实例, 由于平面 1.2 与 2.2 , 1.3 与 2.3 之间不发生接触, 因此, 仅需要计算平面 1.1 与 2.1 , 柱面 2.4 与 3.1 之间的接触变形, 在求得 变形后的非理想表面后, 便能够得到对应的多面体 模型。以平面配合的接触为例, 此处用到的计算参
数为: 两零件的弹性模量均为 $69 \mathrm{GPa}$, 泊松比均为 0.34 , 计算时的外力大小为 $3 \mathrm{kN}$ 。平面 1.1 与 2.1 对应的初始非理想表面与变形后非理想表面如 图 19 所示, 同时, 与平面特征相关的几组多面 体模型如图 20 所示。结果表明是否考虑表面形 貌与受力变形对平面特征的多面体模型有很大 影响。

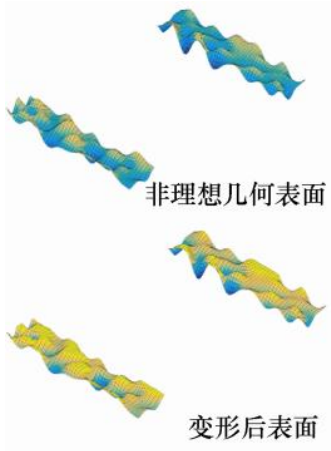

(a) $\mathrm{S} 11$

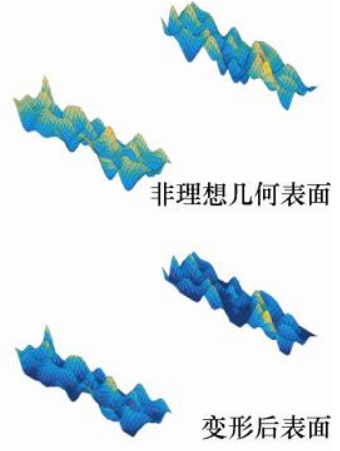

(b) $\mathrm{S} 21$
图 19 变形后的非理想平面

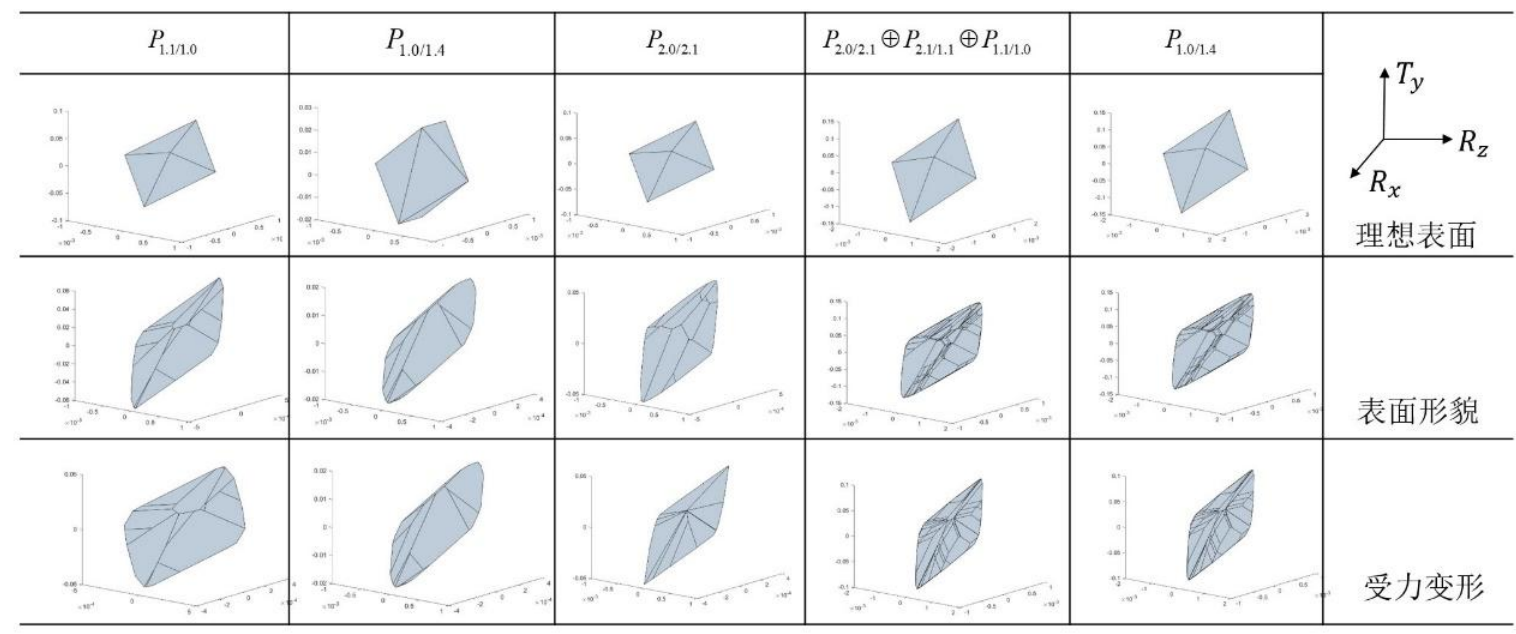

图 20 与平面特征相关的多面体模型 
为了对比表面形貌与受力变形对装配精度分析结 果的影响, 这里对三类情况进行对比, 即: 采用 理想表面模型且不考虑受力变形、考虑表面形貌 但不考虑受力变形、综合考虑表面形貌与受力变 形。不同情况下求得的功能要求多面体如图 21 所 示。图 21 中多面体的形状表明考虑表面形貌与受 力变形可以得到更为精确的装配精度分析结果。 此外, 三种情况下多面体 $P_{3.1 / 1.4}$ 的计算时间分别 为 $12.099 \mathrm{~s}, 37.013 \mathrm{~s}$ 与 $37.297 \mathrm{~s}$, 计算效率能够 满足精度分析要求。

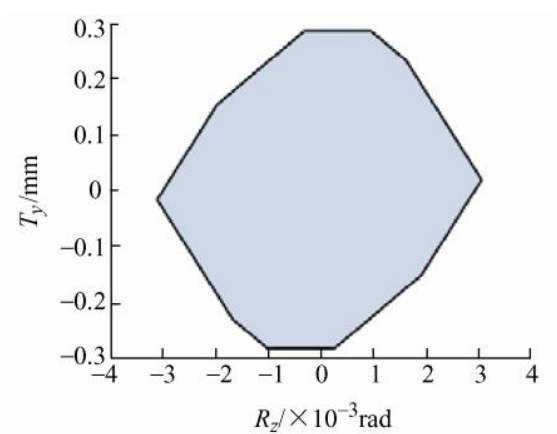

(a) 理想表面

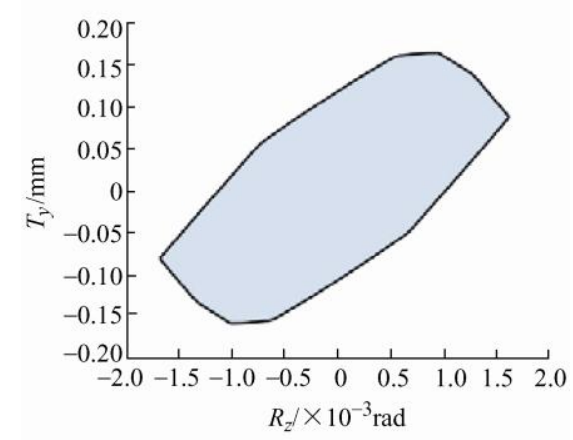

(b) 表面形貌

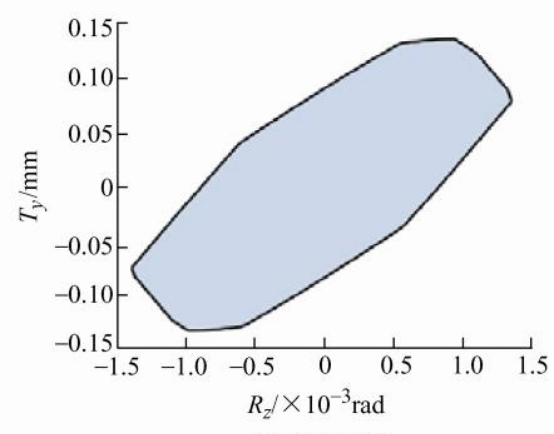

(c) 受力变形

图 21 不同计算条件下的多面体模型 $P_{3.1 / 1.4}$

\section{4 结果对比与分析}

为对比不同计算条件下得到的功能要求多面体 $P_{3.1 / 1.4}$ 的大小关系, 这里将图 21 中的三个多面体放 置于同一坐标系下, 如图 22 所示。

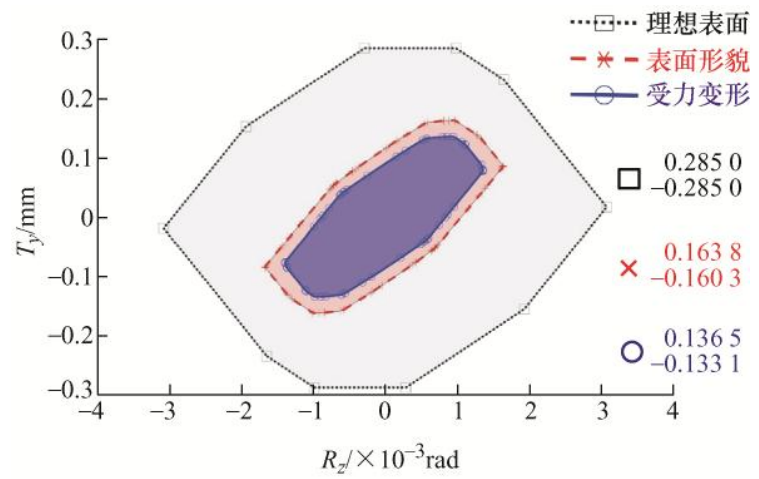

图 22 不同计算条件下 $P_{3.1 / 1.4}$ 对比示意图

由图 22 可以清晰地判断出不同情况下功能要 求多面体 $P_{3.1 / 1.4}$ 的大小关系。具体来说, 对于传统 多面体模型分析方法得到的结果, 点 $M$ 相对于底座 的平动变化范围为 $-0.2850 \sim 0.2850 \mathrm{~mm}$; 当考虑表 面形貌时，该变动范围为 $-0.1603 \sim 0.1638 \mathrm{~mm}$, 相 比于前者, 该范围缩减了大约 $42.5 \%$; 当进一步考 虑受力变形时, 变动范围进一步缩减了 $16.7 \%$, 变 为 $-0.1331 \sim 0.1365 \mathrm{~mm}$ 。以上结果定量体现了表面 形貌与受力变形对于装配精度分析结果的影响, 提 供了更为准确的公差分析结果, 有助于提高装配精 度。上述变动范围的缩减是因为: (1) 对于几何多面 体, 表面形貌与受力变形实际上使得几何特征所允 许的变动范围有所减小, 无法达到传统多面体模型 中允许变动范围的极限值; (2) 对于接触多面体, 非 理想表面能够计算接触表面的实际接触状态, 受力 变形的考虑使得接触状态发生了变化, 而传统多面 体模型则将接触行为简单定义为理想表面的旋转与 平移。因此, 考虑表面形貌与受力变形的装配精度 分析方法能够得到更加准确的计算结果。

\section{5 结论}

（1）采用基函数线性叠加方法对形状误差进行 建模建, 并通过形状误差与基于小位移旋量表达的 定位/定向误差的叠加, 生成了常见几何表面类型的 非理想表面模型, 为装配精度分析过程提供了更为 贴近实际的数学模型, 提高了分析结果的准确性。

（2）基于共轭梯度-快速傅里叶变换方法，实现 了非理想配合矩形平面、圆环面与柱面在载荷作用 下的接触变形计算, 该方法实现了表面形貌与受力 变形的耦合计算, 并兼具准确性与高效性。

(3) 综合考虑表面形貌与受力变形, 对传统多 面体模型进行改进，通过模型间的闵科夫斯基和与 求交运算, 实现了综合考虑表面形貌与受力变形的 装配精度分析, 提高了装配精度分析的准确性, 并 
为公差设计提供指导。

\section{参 考 文 献}

[1] 刘检华, 孙清超, 程晖, 等. 产品装配技术的研究现状、 技术内涵及发展趋势 [J]. 机械工程学报, 2018, 54(11): $2-28$.

LIU Jianhua, SUN Qingchao, CHENG Hui, et al. The state-of-the-art, connotation and developing trends of the products assembly technology[J]. Journal of Mechanical Enginering, 2018, 54(11): 2-28.

[2] 孛朝旺, 杨志宏, 王林博, 等. 尺寸工程技术综述与展 望[J]. 计算机集成制造系统，2014，20(3)：464-470.

BO Chaowang, YANG Zhihong, WANG Linbo, et al. Review and outlook of dimensional engineering[J]. Computer Integrated Manufacturing Systems, 2014, 20(3): 464-470.

[3] REQUICHA A A G. Toward a theory of geometric tolerancing $[\mathrm{J}]$. International Journal of Robotics Research, 1983, 2(4): 45-60.

[4] DESROCHERS A, RIVIERE A. A matrix approach to the representation of tolerance zones and clearances[J]. International Journal of Advanced Manufacturing Technology, 1997, 13(9): 630-636.

[5] WITTWER J W, CHASE K W, HOWELL L L. The direct linearization method applied to position error in kinematic linkages $[\mathrm{J}]$. Mechanism and Machine Theory, 2004, 39(7): 681-693.

[6] BOURDET P, MATHIEU L, LARTIGUE C, et al. The concept of the small displacement torsor in metrology[J]. Advanced Mathematical Tool in Metrology, 1996, 40: 110-122.

[7] DAVIDSON J K, MUJEZINOVIC A, SHAH J J. A new mathematical model for geometric tolerances as applied to round faces[J]. Journal of Mechanical Design, 2002, 124(4): 609-622.

[8] HOMRI L, TEISSANDIER D, BALLU A. Tolerance analysis by polytopes: Taking into account degrees of freedom with cap half-spaces[J]. Computer-Aided Design, 2015, 62: 112-130.

[9] SCHLEICH B, ANWER N, MATHIEU L, et al. Skin model shapes: A new paradigm shift for geometric variations modelling in mechanical engineering $[\mathrm{J}]$. Computer-Aided Design, 2014, 50: 1-15.

[10] SCHLEICH B, WARTZACK S. Evaluation of geometric tolerances and generation of variational part representatives for tolerance analysis[J]. International Journal of Advanced Manufacturing Technology, 2015, 79(5-8): 959-983.

[11] ZHANG M, ANWER N, STOCKINGER A, et al. Discrete shape modeling for skin model representation[J]. Proceedings of the Institution of Mechanical Engineers Part B-Journal of Engineering Manufacture, 2013, 227(B5): 672-680.

[12] 左富昌, 张之敬, 金金金. 考虑平面形状误差的配合表面 接触状态研究 $[J]$. 兵工自动化, 2012(9): 32-37.

ZUO Fuchang, ZHANG Zhijing, JIN Xin. Research on contact state of mating surfaces taking into account plane form error[J]. Ordnance Industry Automation, 2012(9): 32-37.

[13] JBIRA I, TLIJA M , LOUHICHI B , et al. $\mathrm{CAD} /$ Tolerancing integration: Mechanical assembly with form defects[J]. Advances in Engineering Software, 2017, 114: $312-324$

[14] 张体广, 张发平, 阎艳, 等. 基于数据配准的零件精密 装配最佳接触状态研究 [J]. 兵工学报, 2018, 39(1): 127-136.

ZHANG Tiguang, ZHANG Faping, YAN Yan, et al. Research on the best contact state of precision assembly based on data registration[J]. Acta Armamentarii, 2018, 39(1): $127-136$.

[15] 穆晓凯, 孙清超, 孙克鹏, 等. 基于载荷作用的柔性体 三维公差建模及精度影响分析 [J]. 机械工程学报, 2018, 54(11): 39-48.

MU Xiaokai, SUN Qingchao, SUN Kepeng, et al. Three-dimensional tolerance modeling and precision analysis of flexible body based on the assembly load[J]. Journal of Mechanical Engineering, 2018, 54(11): 39-48.

[16] 刘建永, 乔立红. 一种考虑零件变形的装配误差计算方 法[J]. 计算机集成制造系统，2015，21(1)：94-100.

LIU Jianyong, QIAO Lihong. Calculation method for assembly error with consideration of part deformation[J]. Computer Integrated Manufacturing Systems, 2015, 21(1): 94-100.

[17] DAHLSTROM S, LINDKVIST L. Variation simulation of sheet metal assemblies using the method of influence coefficients with contact modeling $[\mathrm{J}]$. Journal of Manufacturing Science and Engineering-Transactions of the ASME, 2007, 129(3): 615-622.

[18] JAYAPRAKASH G, SIVAKUMAR K, THILAK M. FEA compliant parametric tolerance allocation of 
mechanical assembly using neural network and differential evolution algorithm[J]. International Journal of Computer Integrated Manufacturing, 2012, 25(7): 636-654.

[19] KORBI A , TLIJA M , LOUHICHI B , et al. $\mathrm{CAD} /$ tolerancing integration : $\mathrm{A}$ new approach for tolerance analysis of non-rigid parts assemblies[J]. The International Journal of Advanced Manufacturing Technology, 2018, 98(5): 2003-2013.

[20] CORRADO A, POLINI W. FEA integration in the tolerance analysis using skin model shapes[J]. Procedia CIRP, 2018, 75: 285-290.

[21] BEMPORAD A, PAGGI M. Optimization algorithms for the solution of the frictionless normal contact between rough surfaces $[\mathrm{J}]$. International Journal of Solids and Structures, 2015, 69-70: 94-105.

[22] PUTIGNANO C, CARBONE G. A review of boundary elements methodologies for elastic and viscoelastic rough contact mechanics[J]. Physical Mesomechanics, 2014, 17(4): 321-333.

[23] 王文中, 胡元中, 王慧. 基于快速傅里叶变换和共轭梯 度法求解干接触问题 [J]. 机械工程学报, 2006, 42(7):
14-18.

WANG Wenzhong, HU Yuanzhong, WANG Hui. Numerical solution of dry contact problem based on fast Fourier transform and conjugate gradient method[J]. Journal of Mechanical Engineering, 2006, 42(7): 14-18. [24] YASTREBOV V A, ANCIAUX G, MOLINARI J F. From infinitesimal to full contact between rough surfaces: Evolution of the contact area[J]. International Journal of Solids and Structures, 2015, 52: 83-102.

[25] DELOS V, ARROYAVE-TOBÓN S, TEISSANDIER D. Model reduction in geometric tolerancing by polytopes[J]. Computer-Aided Design, 2018, 100: 69-78.

作者简介: 刘检华, 男, 1977 年出生, 博士, 教授, 博士研究生导师。 主要研究方向为产品装配与检测技术。

E-mail: jeffliu@bit.edu.cn

张志强, 男, 1993 年出生, 博士, 工程师。主要研究方向为装配精度分 析技术。

E-mail: ericzhangbit@163.com

夏焕雄(通信作者), 男, 1987 年出生, 博士, 副研究员。主要研究方向 为多相流-多场耦合建模仿真、胶粘连接、精密装配。

E-mail: hxia@bit.edu.cn

巩浩, 男, 1992 年出生, 博士后。主要研究方向为螺纹高可靠性连接、 精密装配。

E-mail: gonghao0220@163.com

邵楠, 男, 1994 年出生, 博士研究生。主要研究方向为公差分析技术。 E-mail: iamshaonan@126.com 